\title{
Attractors: Nonstrange to Chaotic
}

\author{
Robert L. V. Taylor \\ The College of Wooster \\ rtaylor11@wooster.edu \\ Advised by Dr. John David \\ The College of Wooster \\ jdavid@wooster.edu
}

\begin{abstract}
The theory of chaotic dynamical systems can be a tricky area of study for a non-expert to break into. Because the theory is relatively recent, the new student finds himself immersed in a subject with very few clear and intuitive definitions. This paper aims to carve out a small section of the theory of chaotic dynamical systems - that of attractors - and outline its fundamental concepts from a computational mathematics perspective. The motivation for this paper is primarily to define what an attractor is and to clarify what distinguishes its various types (nonstrange, strange nonchaotic, and strange chaotic). Furthermore, by providing some examples of attractors and explaining how and why they are classified, we hope to provide the reader with a good feel for the fundamental connection between fractal geometry and the existence of chaos.
\end{abstract}

\section{INTRODUCTION}

$\mathbf{T}$ HE theory of dynamical systems is an extremely broad area of study. It exists as a science between many different disciplines and can be applied at all levels - from the cosmic to the quantum. Out of the many dynamical systems we have discovered and developed, possibly the most interesting are those that exhibit attracting behavior. In such systems, all initial states tend to evolve towards a single final state, or perhaps a set of final states. These models contain what are known as attractors.

The attractor is much more than simply another phenomenon of nature. It happens to tie together two very closely related mathematical fields: chaos theory and fractal geometry. Anyone who has studied either fractal geometry or chaos theory should know that the two subjects are connected at a very deep level. Furthermore, this connection is not at all easily grasped. By investigating the various types of attractors and exploring the notion of strangeness, this paper hopes to provide an example of how fractals and chaotic dynamics are related.

In Section II, we briefly discuss some of the mathematical tools used to identify and describe the various types of attractors. These concepts are developed from the ground up: first we introduce fractional dimension, used to identify fractal geometry; then the Lyapunov spectrum, used to identify chaotic behavior; and lastly we define what it means to be an attractor and, in particular, a strange attractor. It is a common misconception among non-experts that the term strange attractor is simply another way of saying chaotic attractor. We will show why this is not necessarily true. In Section III, we apply these ideas by classifying and identifying properties of several example attractors.

It is assumed that the reader has a rudimentary understanding of dynamical systems. For example, the reader should be familiar with basic concepts concerning initial conditions, orbits, and the differences between real and discrete systems.

\section{Mathematical Concepts}

\section{A. Fractals and Dimension}

Simply put, a fractal is a geometric object that is selfsimilar on all scales. This somewhat vague description applies to a very wide variety of geometric objects found in both the abstract and natural worlds. Coastlines, mountains ranges, and trees are some typical examples of natural objects with fractal properties. Although this paper is primarily concerned with fractals as they relate to mathematical dynamics, it is important to keep in mind that many times these ideas are inspired by phenomena we observe in nature.

Since the term fractal refers to a very generalized class of geometric object, it is probably best defined by identifying some of its typical characteristics. Whenever we refer to a set $F$ as a fractal, we typically have the following in mind [3]:

- Often $F$ has some form of self-similarity, perhaps approximate or statistical.

- $F$ has a fine structure - i.e. detail at arbitrarily small scales.

- $F$ is too irregular to be described in traditional geometrical language, both locally and globally.

- Usually, the fractional dimension of $F$ is greater than its topological dimension.

- In most cases of interest, $F$ is defined in a very simple way, perhaps recursively.

Of all the charateristics listed above, perhaps the most relavent to this paper is that which refers to the fractional dimension of a set. Just as some geometric 
objects can be 2-dimensional or 3-dimensional, there can also exist objects that have dimensions of $\frac{1}{2}, \frac{7}{4}$, or 2.43 . For example, it is very clear to us that in Euclidean space, a line segment has a dimensionality of 1 , a solid square has a dimensionality of 2 , and a solid cube has a dimensionality of 3 . However, perhaps less clear are the reasons why this is true. As it turns out, each of these geometric objects is $n$-dimensional because it completely fills in a section of $\mathbb{R}^{n}$. In other words, a line segment is 1-dimensional because we can measure its length, a solid square is 2-dimensional because we can measure its area, and a solid cube is 3-dimensional because we can measure its volume.

Now consider another kind of geometric object - one that is only able to partially fill in a section of space. Suppose we have an extremely curvy line drawn on a plane. One might say that since it is a line, and thus has length, it must be 1-dimensional. But imagine that this curve is special in that it is so curvy that it also partially fills in some amount of area. Such a curve is niether 1-dimensional nor 2-dimensional. In this case, the curve has a fractional dimension $1<d<2$. The Koch snowflake is an example of such a curve.

Definition II.1. A set of points is said to be fractal if its dimension is non-integer.

Here it is important to point out that a round integer dimension does not necessarily imply that a set is nonfractal. Even sets that have integer dimensions can have fractal properties. For such special cases we must develop alternative methods to quantify the hidden fractal geometry. In Section III-C, we encounter a fractal set that has an integer dimension.

There exist many different ways of estimating dimension, and each has its own advantages and disadvantages. One measurement that is found very often in introductory liturature on fractals is the similarity dimension. When dealing with very simple fractals, such as the Koch snowflake, this measure can easily be calculated by hand. Unfortunately, for the types of fractal sets that we encounter in this paper, the similarity dimension is not particularly useful. Instead, we use the KaplanYorke dimension, denoted $D_{K Y}$, because it lends itself well to dynamical systems. Since it is closely related to the Lyapunov spectrum, it is explained at the end of the next section.

The similarity dimension and the Kaplan-Yorke dimension, combined with other dimensional measures such as the capacity, pointwise, correlation, and information dimensions, provide mathematicians with a wide variety of tools to choose from when investigating fractal sets. Luckily, when it comes to calculating fractional dimension of strange attracting sets - a main focus of this paper - these various measures of dimension are considered roughly equivalent [7].

\section{B. The Lyapunov Spectrum}

The Lyapunov exponent measures the sensitivity of a dynamical system to small changes in initial conditions, and is used primarily for identifying chaos. If two orbits start close to one another in a chaotic system, then they will tend to move away from each other exponentially. We can write this idea as

$$
\frac{d}{d_{0}}=e^{\lambda\left(t-t_{0}\right)}
$$

where $d_{0}$ is the initial displacement between a starting point and a nearby neighbor at initial time $t_{0}$. The variable $d$ is the displacement at time $t>t_{0}$. The $\lambda$ is the Lyapunov exponent. We can see that if $\lambda>0$, then $\frac{d}{d_{0}}$ grows exponentially with the passage of time; if $\lambda<0$, then $\frac{d}{d_{0}}$ shrinks to zero. If $\lambda=0$, then the change in displacement over time is non-exponential.

Please note that use of $e$ as the constant is somewhat arbitrary. Traditionally, if the system is continuous, we use base $e$; if the system is a discrete mapping, we use base 2 .

By solving the equation above for $\lambda$ we get

$$
\lambda=\frac{1}{t-t_{0}} \ln \left|\frac{d}{d_{0}}\right|
$$

This equation only provides a means for calculating $\lambda$ for two specific neighboring points over a specific interval of time. Since we want to be able to approximate the Lyapunov exponent for an entire dynamic system, taking a single measurement, as shown above, is not sufficient. Thus, to approximate the true value of $\lambda$ for an entire system, we need to average it over many different neighborhoods.

Definition II.2. If the displacement between the $i$-th point and a neighboring point at time $t_{i}$ is $d_{i}$, and the initial displacement between the two points is $d_{0 i}$ at time $t_{0 i}$, then the Lyapunov exponent is defined as

$$
\lambda=\lim _{n \rightarrow \infty} \frac{1}{n} \sum_{i=1}^{n} \frac{1}{\left(t_{i}-t_{0 i}\right)} \ln \left|\frac{d_{i}}{d_{0 i}}\right|
$$

This definition for the Lyapunov exponent is convenient because it allows us to approximate $\lambda$ for just about any dynamical system using a computer. By randomly choosing a large number of neighboring pairs of points in a dynamical system, and observing their relative movement to one another, a value for $\lambda$ can easily be calculated. However, this is not the only way in which $\lambda$ can be defined - there are several other similar definitions which are much more analytical in nature. These varying definitions can be found in most introductory literature on chaos theory.

Now, to further complicate matters, most dynamical systems have more than one Lyapunov exponent. If a system is defined in $n$-dimensions, then that system has $n$ Lyapunov exponents, one for each of its dimensions. Combined, this set of exponents is referred to as the Lyapunov spectrum. The spectrum is typically ordered 
from largest exponent to smallest. To find the complete spectrum, we must calculate $\lambda_{i}$ for small displacements in the $i$-th dimension, for each $1 \leq i \leq n$.

Definition II.3. If $\lambda_{1} \geq \lambda_{2} \geq \cdots \geq \lambda_{n}$ are the Lyapunov exponents for a dynamical system in $\mathbb{R}^{n}$, then the Lyapunov spectrum is the set $\left\{\lambda_{1}, \lambda_{2}, \ldots, \lambda_{n}\right\}$.

We can identify chaos with the following criterion:

$$
\begin{aligned}
& \lambda_{1}>0 \Leftrightarrow \text { chaotic } \\
& \lambda_{1} \leq 0 \Leftrightarrow \text { non-chaotic }
\end{aligned}
$$

Not only can we use the Lyapunov spectrum to identify chaos in a dynamical system, but we can also use it to approximate the fractional dimension of the set of points on which the dynamical system lives. With the help of the Lyapunov spectrum, it is a simple matter to compute the Kaplan-Yorke dimension.

Definition II.4. If $\lambda_{1} \geq \lambda_{2} \geq \cdots \geq \lambda_{n}$ are the Lyapunov exponents for a dynamical system in $\mathbb{R}^{n}$ and $j$ is the largest integer for which $\lambda_{1}+\lambda_{2}+\cdots+\lambda_{j} \geq 0$, then the Kaplan-Yorke dimension is given by

$$
D_{K Y}=j+\frac{\lambda_{1}+\lambda_{2}+\cdots+\lambda_{j}}{\left|\lambda_{j+1}\right|}
$$

\section{Computing Lyapunov Exponents}

A problem regarding the Lyapunov exponent quickly arises when studying dynamical systems. Since these systems tend to be bounded, they usually exhibit orbits that diverge and then merge back onto each other. Calculating exponential divergence becomes much trickier due to this folding and stretching. When simulating the orbits of two points, we must be careful to simulate only as far as needed to see them initially diverge, then stop when folding back occurs.

So, instead of calculating the Lyapunov exponent by averaging the divergence over a large number of pairs of orbits, as the definition of Lyapunov exponents in the previous section indicates, a slightly altered method is used. Consider the following algorithm, adapted from [10].

1. Pick a random point. This set of initial conditions generates a single reference orbit in our system, which we will compare with other nearby orbits.

2. Select a nearby point. The new point and the reference point should be seperated by some small initial displacement $d_{0}$. The point should be displaced in the direction of the dimension for which we are calculating a Lyapunov exponent.

3. Advance both orbits a small amount and calculate the new seperation $d_{1}$. In the case of a discrete mapping, we advance the orbits by computing one iteration. For continuous models, we have to choose some small time interval $t_{\text {step }}$ to move the model forward by. Either way, we then calculate $d_{1}$ by finding the norm of the difference vector between the two points.
4. Calculate the local Lyapunov exponent. In the continuous case, this is given by

$$
\frac{1}{t_{\text {step }}} \ln \left|\frac{d_{1}}{d_{0}}\right|
$$

For discrete mappings, we have

$$
\log _{2}\left|\frac{d_{1}}{d_{0}}\right|
$$

5. Readjust the second orbit. This should be done so thats its displacement from the reference orbit is again $d_{0}$, but in the same direction as $d_{1}$. See Figure 1.

6. Repeat steps (4-6) $n$ times to produce an average Lyapunov exponent. The value of $n$ needs to be large enough for us to see the average exponent converge to a single value. Thus, for the continuous case,

$$
\lambda=\frac{1}{n\left(t_{\text {step }}\right)} \sum_{i=1}^{n} \ln \left|\frac{d_{i}}{d_{0}}\right|
$$

For discrete mappings, we have

$$
\lambda=\frac{1}{n} \sum_{i=1}^{n} \log _{2}\left|\frac{d_{i}}{d_{0}}\right|
$$

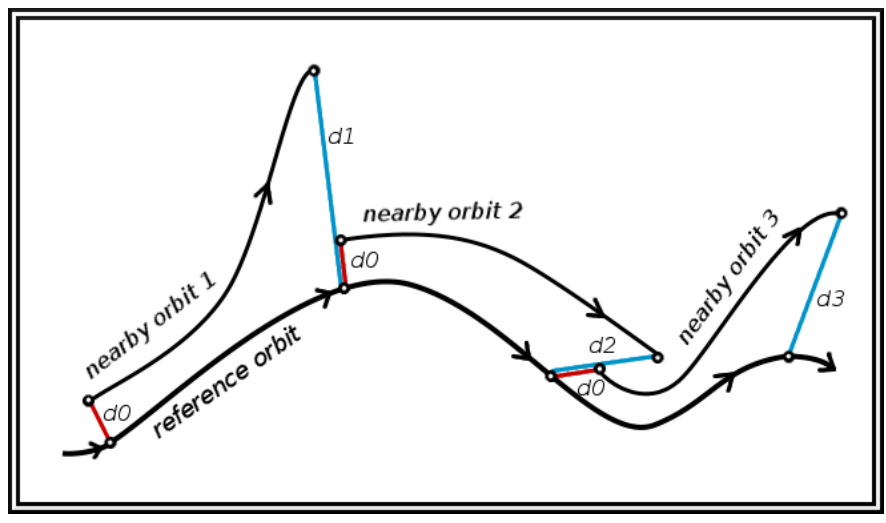

Fig. 1. The algorithm for calculating a Lyapunov exponent of an attractor. First, a reference point and a nearby point are chosen (the distance between them is $d_{0}$ ). They are then briefly allowed to deviate from (or converge to) one another along their respective orbits. After which, their seperation is recorded $\left(d_{1}\right)$. Then, a new point is chosen near to the reference orbit - one in the same direction as the current position of the first neighbor, but displaced from the reference orbit by $d_{0}$. Again, they are allowed to deviate and a second seperation is recorded $\left(d_{2}\right)$. This process is repeated $n$ times to find the set $\left\{d_{1}, d_{2}, \ldots, d_{n}\right\}$. An approximation of $\lambda$ can then be calculated with Equation 9 or 10 .

To calculate the entire Lyapunov spectrum for a dynamical system, we need to go through these steps for each of the system's dimensions. So, in the case of a 2-dimensional system, we must use the algorithm to calculate a $\lambda$ for small dispacements along both the $x$ axis and the $y$-axis. Figure 2 demonstrates the calculation of the Lyapunov spectrum for one of the dynamical systems discussed in Section III, the Rayleigh attractor. 


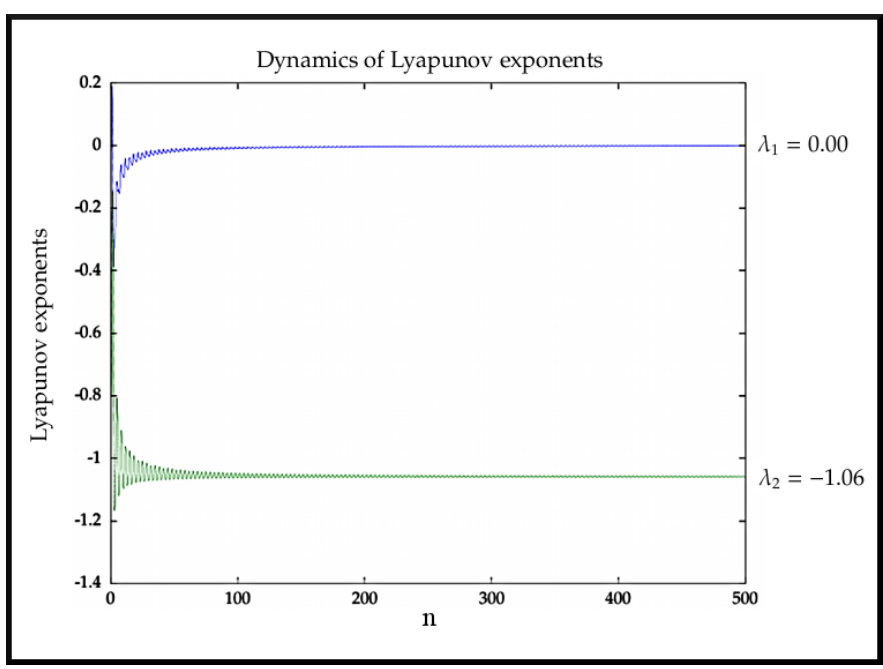

Fig. 2. The calculation of the Lyapunov spectrum for the Rayleigh dynamical system (Section III-B). The system exists in two dimensions - so it has two Lyapunov exponents. As $n$ increases in Equation 9, we see that each $\lambda$ converges to a single value. The Lyapunov spectrum is found to be $\left\{\lambda_{1}=0, \lambda_{2}=-1.06\right\}$. Since both exponents are nonpositive, the system is nonchaotic.

\section{Attractors}

The following definition of an attracting set is an adaptation of the rigorous definition given by Milnor [6]. While Milnor's is very generalized and applies in a wide varety of mathematical contexts, the one given here has been simplified to deal only in Euclidean space (i.e., $\mathbb{R}^{n}$ ).

Definition II.5. A set $A \subset \mathbb{R}^{n}$ will be called an attracting set if the following two conditions are true:

1) The basin of attraction $B(A)$, consisting of all points whose orbits converge to $A$, has strictly positive measure.

2) For any closed proper subset $A^{\prime} \subset A$, the set difference $B(A) \backslash B\left(A^{\prime}\right)$ also has strictly positive measure.

Since we are restricted to $\mathbb{R}^{n}$, it should be easy to gain an intuitive understanding of what the above definition of an attracting set means. The first condition basically states that our basin of attraction must be, in some sense, tangible. It cannot be a single point or a set of discontinuous points (sets whose measures are zero). Our basin of attraction must consist of some sort of $n$ dimensional interval. If we consider the space $\mathbb{R}^{2}$, the first condition states that any basin of attraction must have some positive area. If we consider the space $\mathbb{R}^{3}$, any basin of attraction must have some positive volume.

The second condition is slightly more nuanced. It says that if we were to change our attracting set at all, then the measure of our basin of attraction would also change. In $\mathbb{R}^{2}$, for example, if we were to remove a single point from the attracting set, the basin of attraction would lose area.

Alternatively, if we remove points from $A$ by defining a new, closed set $A^{\prime} \subset A$, and it turns out that the basin does not significantly change (the set difference $B(A) \backslash B\left(A^{\prime}\right)$ has measure zero), then the implication would be that the removed points were not actually attracting a significant portion of the basin. In this case, $A$ would not be considered an attracting set.

Milnor gives the a nice explanation of the two conditions for an attracting set [5]: "the first condition says that there is some positive possibility that a randomly chosen point will be attracted to $A$, and the second says that every part of $A$ plays an essential role."

In order for an attracting set to be considered an attractor, it must satisfy a third condition [6]:

Definition II.6. An attractor is an attracting set that contains a dense orbit.

The existence of a dense orbit on an attracting set means that there is at least one orbit which either passes through, or gets infinitely close to, every point in the attracting set. This condition ensures that the attracting set is not simply the union of two smaller attracting sets. If the attracting set were the union of two smaller attracting sets, then there could not possible exist a single orbit that is dense on both smaller sets.

There are three known types of nonstrange attractors: the fixed point attractor, the limit cycle attractor, and the torus attractor [2]. These are nonfractal attractors, the behavior of which can be accurately predicted. If we were given the initial conditions of some orbit on one of these simple attractors, we would be able to use that information to predict the orbit with some degree of confidence. We consider two nonstrange attractors in Sections III-A and III-B.

Each of the three types of nonstrange attractor is associated with a specific type of orbit behavior. The fixed point is associated with attractors whose orbits approach an equilibrium state; the limit cycle corresponds to periodic motion; and the torus corresponds to quasiperiodic motion (almost, but not quite periodic) [7].

\section{E. Strange Attractors}

The term strange is most often used as a name for attractors that exhibit chaotic behavior - i.e., sensitivity to initial conditions. Though indeed true, this use of the term is somewhat misleading. It is important to clarify that strangeness is not dependent on the existence of chaos. Though attractors showing extreme sensitivity to initial conditions are indeed strange, strange attractors need not be chaotic. Consider the following definition of strangeness.

Definition II.7. An attractor is strange if its attracting set is fractal in nature.

While the term chaotic is meant to convey a loss of information or loss of predictability, the term strange is meant to describe the unfamiliar geometric structure on which the motion moves in phase space [7].

In a chaotic regime, orbits on an attractor are nonperiodic. Thus, any given point in the attracting set is never visited more than once, and there are entire regions of 
points that are never visited. Such sets of points are fractal in nature and usually have non-integer dimension. It follows that if an attractor exhibits chaotic behavior, then it is a strange attractor. Refer to Crutchfield et. al. for detailed figures that illustrate the differences between several well-known chaotic strange and nonstrange attractors [2]. In Section III-D we investigate the Lorenz strange attractor.

Not all strange attractors are chaotic. There exist examples of strange nonchaotic attractors (SNA). These SNA's are distinguished from their chaotic counterparts by their lack of sensitivity to initial conditions. As an example of an SNA, consider the GOPY attractor in Section III-C.

\section{Classifying an Attractor}

In this section, we look at four distinct attractors and consider the problem of identifying some of their properties. We are mainly interested in how to identify their general classification: nonstrange, strange nonchaotic, or strange chaotic. We use Lyapunov exponents to identify chaotic behavior and we use what we know about fractals to identify strangeness. Plots of the orbits in each model also help us gain an intuitive feel for the type of attracting set.

\section{A. Buckling Column Model}

The buckling column model is defined by the system of differential equations [1]:

$$
\begin{aligned}
& \dot{x}=y \\
& \dot{y}=\frac{-1}{m}\left(a x^{3}+b x+c y\right)
\end{aligned}
$$

The buckling column system has the interesting property in that under slightly different parameter conditions, two different types of attracting set can appear. For example, when $a=20, b=10, c=5$, and $m=10$, we find a fixed point attractor (Figure 3). However, if we change $b=10$ to -10 , then we get a two fixed point attracting set - which is not a single attractor at all, since it consists of two distinct basins of attraction, each being pulled by a different attracting point (Figure 4).

By using the algorithm of Section II-C, we can easily approximate the Lyapunov spectrum of the fixed point attractor of the buckling column system $(b=10)$. We find $\lambda_{1,2}=-0.25$ for displacements in both the $\dot{x}$-axis and the $x$-axis. In fact, negative exponents should be expected since all observable orbits in all directions seem to be converging to a single point: the origin. Since there are no positive Lyapunov exponents, the system is nonchaotic.

Since the attractor seems to consist of a single point, we would expect the fractal dimension of the attracting set to be 0 . Indeed,

$$
D_{K Y}=0 .
$$

Thus, the buckling column attractor is nonstrange, since it does not exhibit a fractal structure.

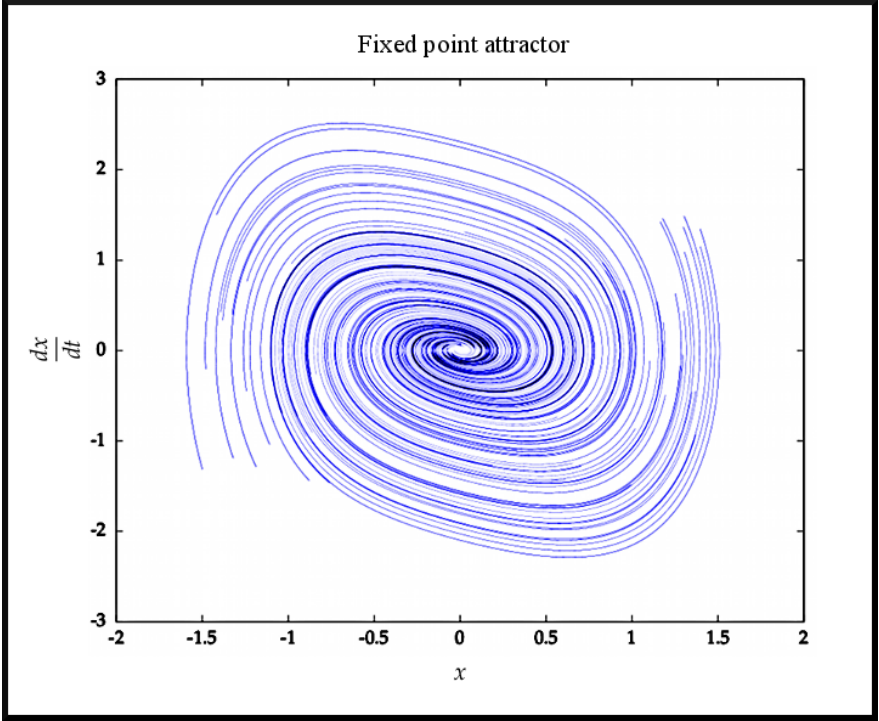

Fig. 3. Phase portrait of the buckling column model with one fixed point attractor at origin. In this case, $a=20, b=10, c=5$, and $m=10$ in Equation 12. From this plot we can see a highly predictable structure, with all orbits cycling toward the origin. This attractor is nonstrange.

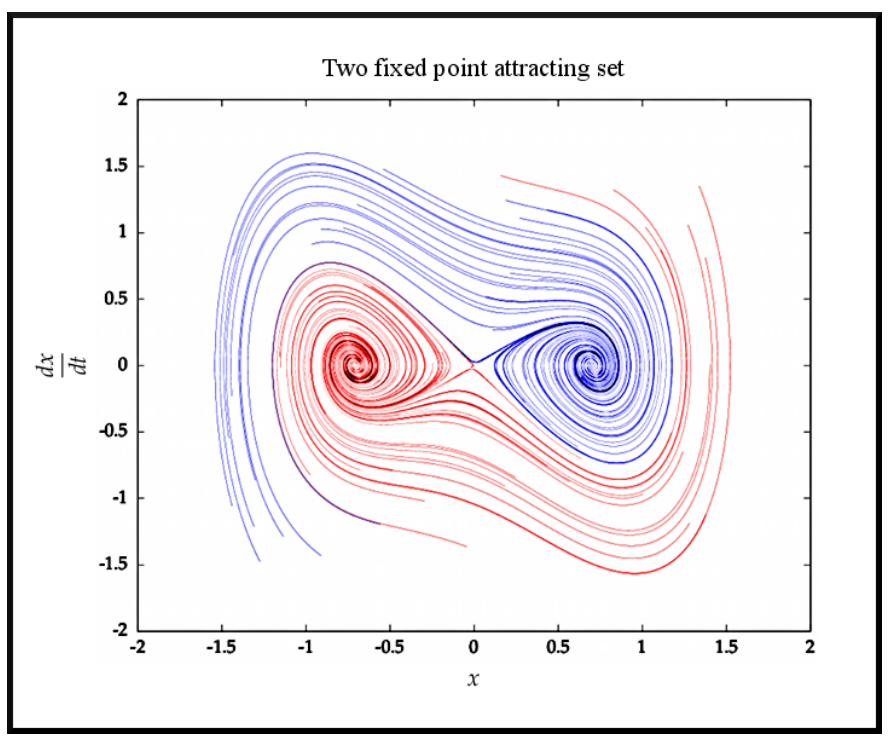

Fig. 4. Phase portrait of the buckling column model with a two fixed point attracting set. Notice that this is not a single attractor as there are two seperate basins of attraction. In this case, $a=20, b=-10, c=5$, and $m=10$ in Equation 12. From this plot we can see a highly predictable structure, with all orbits cycling toward one of the two points. This attracting set is nonstrange.

\section{B. Rayleigh Model}

The Rayleigh model is defined by the system of differential equations [1]:

$$
\begin{aligned}
& \dot{x}=y \\
& \dot{y}=\frac{-1}{c d}\left(x+b y^{3}-a y\right)
\end{aligned}
$$

When the parameters are set as $a=b=c=d=1$, we find ourselves a limit cycle attractor (Figure 5). 
Using the algorithm in Section II-C, we find that the Lyapunov exponents are $\lambda_{1}=0$ and $\lambda_{2}=-1.06$. This particular calculation is illustrated by Figure 2 . Since neither exponent is positive, we conclude that the Rayleigh attractor is indeed nonchaotic. Using the Kaplan-Yorke formula to find the attracting set's fractional dimension, we get

$$
D_{K Y}=1+\frac{0}{|-1.06|}=1
$$

We conclude that the Rayleigh attractor is nonstrange, since it does not exhibit a fractal structure.

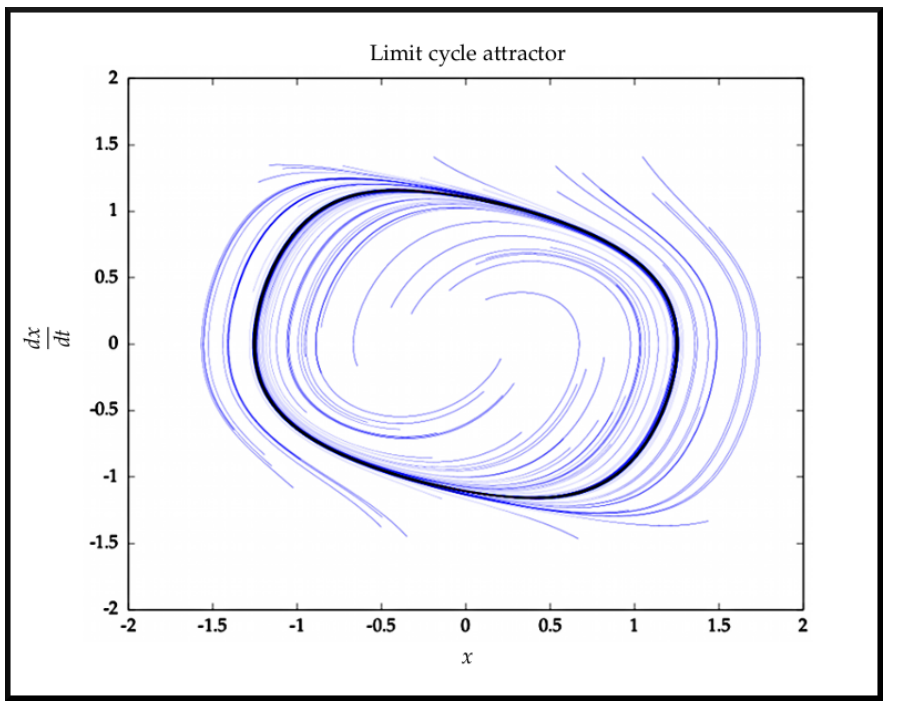

Fig. 5. Phase portrait of the Rayleigh model with a limit cycle attractor. From this plot we can see a highly predictable structure, with all orbits being pulled toward a ring-like attracting set. This attractor is nonstrange.

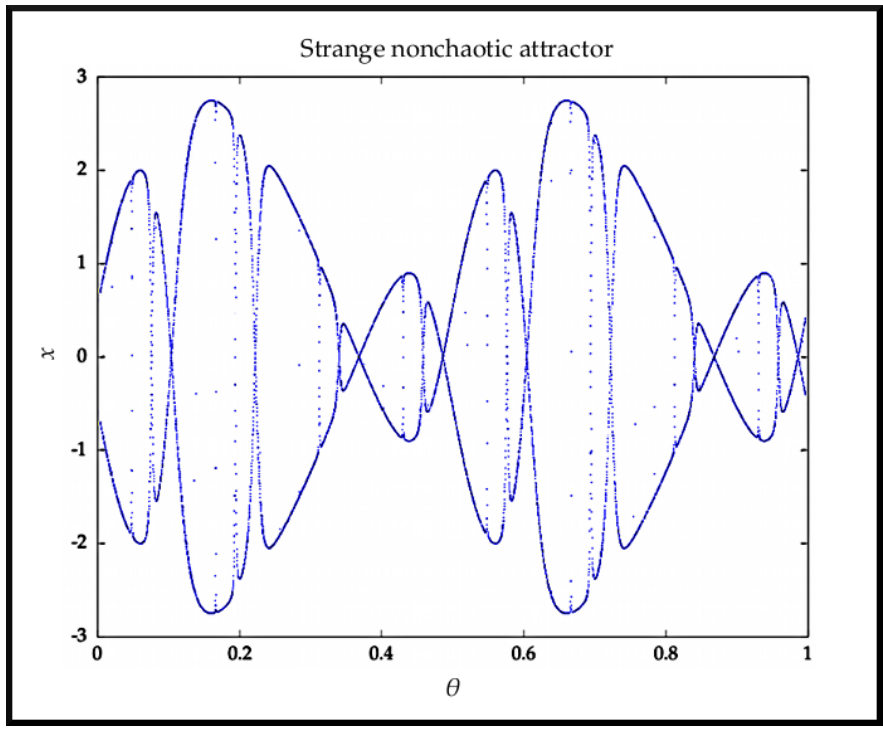

Fig. 6. A single orbit on the GOPY attractor. Notice the interesting structure produced by this discrete mapping. Though it appears that the attracting set consists of looping "curves", it is completely discontinuous. It can be shown that between any two points on the lower "curve" there exists a point on the upper "curve". Furthermore, since $\theta_{n+1}$ is simply $\theta_{n}$ plus an irregular number modulo 1, we know that the orbit is nonperiodic. These facts imply a fractal structure.

\section{GOPY Model}

Grebogi et. al. were the first to show that strange attractors need not be chaotic [4]. It can be shown that the GOPY attractor (unofficially named after the authors) has a fractal structure with nonpositive Lyapunov exponents.

The GOPY system is defined by a discrete mapping in two dimensions:

$$
\begin{aligned}
& x_{n+1}=2 \sigma \tanh \left(x_{n}\right) \cos \left(2 \pi \theta_{n}\right) \\
& \theta_{n+1}=\theta_{n}+\omega \bmod 1
\end{aligned}
$$

We find that a strange attractor appears in the GOPY system when $\sigma=1.5$ and $\omega=\frac{\sqrt{5}-1}{2}$. Notice that $\omega$ is defined as the golden ratio, an irrational number. In fact, $\omega$ can be any irrational number - the strange attractor will appear regardless.

We approximate the Lyapunov spectrum of the GOPY attractor by using the algorithm of Section II-C. We find $\lambda_{1}=0$ for displacements in the $\theta$-axis, and $\lambda_{2}=-1.53$ for displacements in $x$-axis. Thus, the system is nonchaotic because there are no positive Lyapunov exponents.

Here is the tricky part. Showing that the GOPY attractor is fractal is not a simple matter of showing that its fractional dimension is non-integer. Notice that the Kaplan-Yorke dimension is an integer:

$$
D_{K Y}=1+\frac{0}{|-1.53|}=1
$$

We must find another way to show that the GOPY attractor has a fractal structure.

When we consider Figure 6, the attractor's fractalness is not immediately obvious. It simply looks like an interesting curve. But recall that the system is defined by a discrete mapping, so the attractor we are seeing is not a curve at all, but the plot of a collection of iterations. What is more, for almost all of the interval $0 \leq \theta \leq 1$ there are two distinct "curves": one above and one below.

Suppose that between any two points on the upper "curve" there exists a point on the lower "curve" (and vice versa). Then the attractor would have a fine structure (detail at arbitrarily small scales). By demonstrating this property numerically, we can argue that the GOPY attractor has a fractal geometry and is therefore strange.

Since the value of $x$ is bouncing back and forth between the curves for even the smallest of changes in $\theta$, we would expect the maximum slope $\frac{d x}{d \theta}$ between any two points on the attractor to get arbitrarily large as we continue to iterate and compare points. In other words, as the number of iterations approaches infinity, we should be able to find arbitrarily large slope values.

Pikovsky and Feudel apply this concept by introducing a phase sensitivity measure $\Gamma_{N}$ to characterize the strangeness of the GOPY attractor [8]. The following is an adaptation of their definition. 
Definition III.1. Let $\Lambda$ be a set of arbitrary initial points in the GOPY attractor. Define the phase sensitivity

$$
\Gamma_{N}=\min \left\{\max \left\{\left|\frac{x_{n}-x_{0}}{\theta_{n}-\theta_{0}}\right|: 1 \leq n \leq N\right\}:\left(x_{0}, \theta_{0}\right) \in \Lambda\right\}
$$

to be an approximate lower bound on the maximum slope $\frac{d x}{d \theta}$ at any arbitrary point in the attractor after $N$ iterations.

This definition is complex and requires some explanation. Suppose $N=100$ and $\Lambda$ is a set of ten randomly chosen initial points in the GOPY system. For each of the ten initial points, we calculate $N=100$ subsequent points in its orbit and determine the maximum slope that occurs between it and any of its iterations. This results in a set of ten maximum values - the minimum of which is $\Gamma_{N}$, an approximate lower bound on the maximum slope at any arbitrary point in the attractor after $N$ iterations.

If $\Gamma_{N}$ grows indefinitely as $N \rightarrow \infty$, then the attractor has a fractal structure and is strange. Essentially, this is because an unbounded $\Gamma_{N}$ indicates that for any two points on the upper "curve" there exists a point on the lower "curve".

As an additional note, it has been theorized that $\Gamma_{N}$ grows exponentially for strange chaotic attractors, while for strange nonchaotic attractors, $\Gamma_{N}$ grows as a power,

$$
\Gamma_{N}=c N^{\mu}
$$

with $\mu$ typically greater than 1 and $c$ a constant [9].

To show that the GOPY attractor is strange, we calculate $\Gamma_{N}$ for 10 values of $N$, ranging from 10 to $10^{6}$. It is found that the coordinate pairs $\left(N, \Gamma_{N}\right)$ can be approximated by $\Gamma_{N}=(2.1) N^{1.08}$. Since the growth of $\Gamma_{N}$ seems to be unbounded, we have numerical evidence that the GOPY attractor is strange.

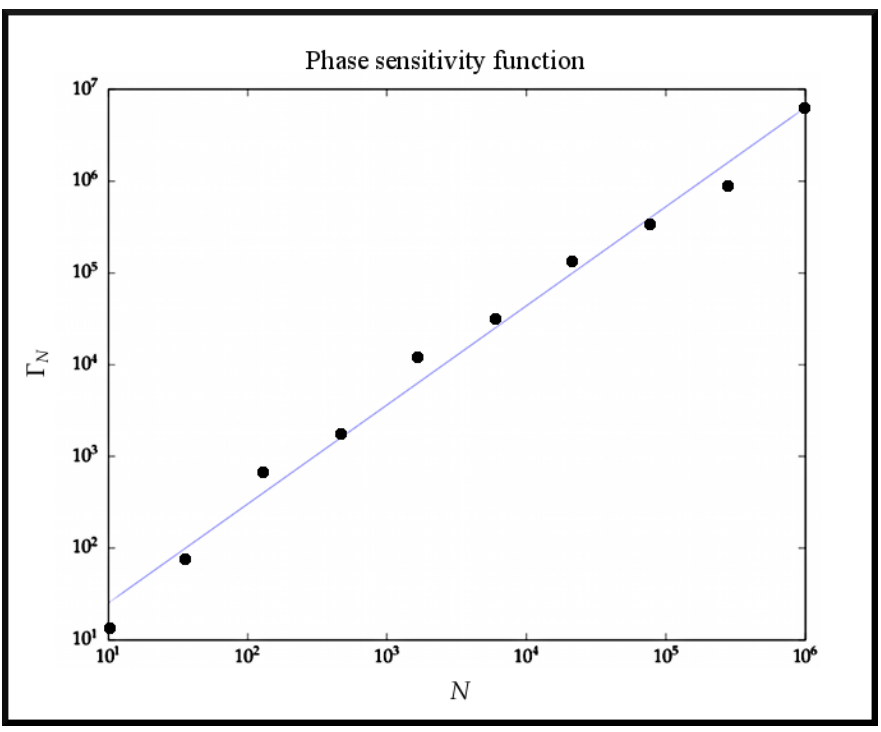

Fig. 7. Ten calculations of $\Gamma_{N}$ for the GOPY attractor ( $\Lambda$ contains 100 randomly chosen initial points). These ten values are approximated by the line $\Gamma_{N}=(2.1) N^{1.08}$. The divergence of $\Gamma_{N}$ shown here indicates that the GOPY attractor has a fractal structure and is strange.

\section{Lorenz Model}

The Lorenz attractor is possibly the most famous strange attractor and is characterized by its unique butterfly shape. It is defined in three dimensions by the system of differential equations:

$$
\begin{aligned}
& \dot{x}=\sigma(y-x) \\
& \dot{y}=x(\rho-z)-y \\
& \dot{z}=x y-\beta z
\end{aligned}
$$

When $\rho=28, \sigma=10$, and $\beta=\frac{8}{3}$, we find that orbits take the general shape shown in Figure 8 below.

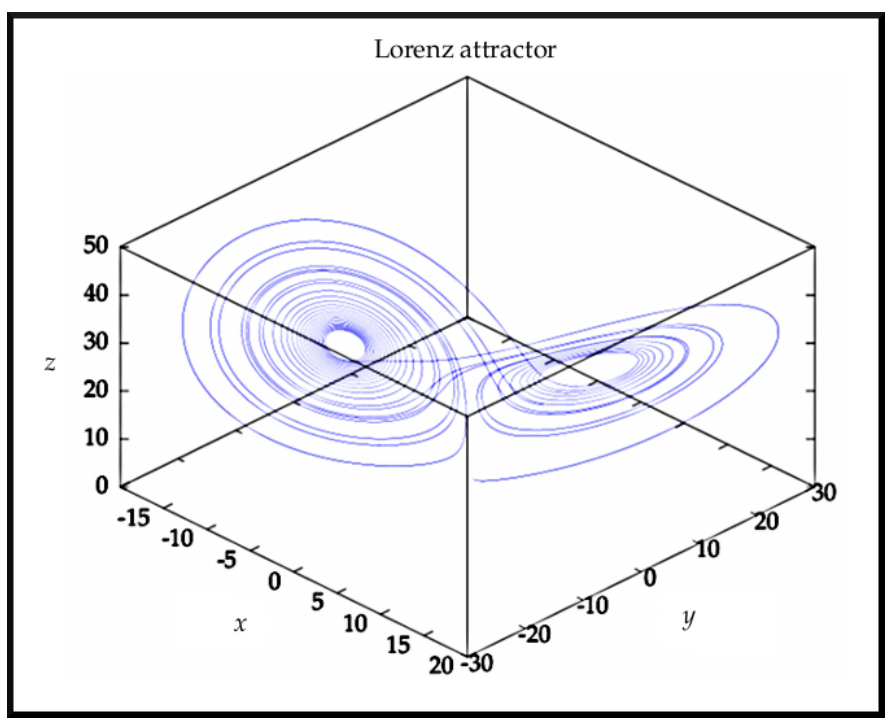

Fig. 8. The Lorenz attractor, possibly the most well-known strange chaotic attractor. As orbits chaotically converge and diverge from one another, a unique butterfly shape emerges. This shape has a fractal geometry.

In order for an orbit in the Lorenz model to exhibit both convergence to the attracting set and exponential divergence from other nearby orbits, it must undergo a continual process of folding and stretching. Indeed, something fundamental about the Lorenz model causes orbits to simultaneously converge to and diverge from each other. This tearing property is part of what causes the attractor's fractal nature, its strangeness.

Of the Lorenz attractor's three Lyapunov exponents, one is positive, one is negative, and one is zero. It has been shown how the signs of the three exponents correspond to the expansion and contraction of orbits along the three axes. The existence of both positive and negative exponents in the spectrum is an important indicator of strangeness. It is further theorized that we can classify attractors based on their Lyapunov spectrum as follows: $(+, 0,-)$ strange; $(0,0,-)$ two-torus; $(0,-,-)$ limit cycle; $(-,-,-)$ fixed point. [11]

By usual method, we can calculate our own Lyapunov spectrum for the Lorenz attractor: $\left\{\lambda_{1}=0.81, \lambda_{2}=\right.$ $\left.0.02, \lambda_{3}=-14.50\right\}$. Although $\lambda_{2}$ did not match up exactly with its expected value of zero, we can still see the mix of positive with negative exponents mentioned above. 
TABLE I

Properties and classifications of the four examples of attractors discussed in Section III.

\begin{tabular}{|c|c|c|c|c|c|}
\hline Attractor & Lyapunov spect & um & Dimension & Phase sens. & Classification \\
\hline Buc & $\lambda_{1}=-0.25$ & & $D_{K Y}=0$ & $n / a$ & nonstrange [fixed point] \\
\hline Ra) & $\lambda_{2}=-1.06$ & & $=1$ & $n / a$ & [limit cycle] \\
\hline G & $\lambda_{2}=-1.53$ & & $D_{K}$ & $\Gamma_{N}=(2.1) N^{1.08}$ & nonchaotic \\
\hline Lorenz & $\lambda_{1}=0.81$ & $\lambda_{3}=-14.50$ & $D_{K Y}=2.06$ & $n / a$ & strange chaotic \\
\hline
\end{tabular}

From the Lyapunov spectrum, we can easily compute the Kaplan-Yorke dimension.

$$
D_{K Y}=2+\frac{0.81+0.02}{|-14.50|}=2.06
$$

Notice that $D_{K Y}$ is a non-integer. Thus, the attracting set has a fractal structure - proof that the attractor is strange. Since $\lambda_{1}>0$, we know that the Lorenz attractor must be both strange and chaotic.

\section{Discussion}

The primary focus of this paper is to gain an understanding of what it means to be an attractor and how this idea relates to chaos and fractals. In particular, we explore the meaning of strangeness. It is a common misconception that the term strange attractor is simply another way of saying chaotic attractor. Though a chaotic attractor is certainly a strange attractor, the reverse is not necessarily true. Instead, the term strange actually refers to the fractal geometry on which chaotic behavior may or may not exist. Indeed, there exist examples of strange attractors which are nonchaotic.

To investigate the connection between fractal geometry and chaotic dynamics, we have introduced several mathematical tools. The Lyapunov spectrum is used primarily to identify sensitivity to initial conditions in a dynamical system. If sensitivity exists, then we say that the system is chaotic. If the attractor in the system has a fractal geometry - as indicated by the Kaplan-Yorke dimension or the phase sensitivity measure $\Gamma_{N}-$ then the attractor is strange. Since it is not always easy to identify chaotic behavior and strangeness analytically, we give special attention to how these tools are used computationally.

To illustrate the various types of attracting behavior, as well as to demonstrate the use of our mathematical tools, several examples of attractors are provided in Section III. Table I summarizes the properties found for the four examples discussed in this section.

The first example is the 2-dimensional Buckling attractor. It has two negative Lyapunov exponents and a dimension of zero, indicating a fixed point attracting set. It is interesting that when the parameters in Equation 12 are varied, two distinct attracting sets can be seen: Figure 3 shows a single fixed point attractor, while Figure 4 shows a two fixed point attracting set with two seperate basins of attraction.

The second example is the 2-dimensional Rayleigh attractor. Its Lyapunov exponents are zero and negative, while its dimension is one. This information tells us that the attractor is a limit cycle. Notice that the next example, the 2-dimensional GOPY attractor, has the same properties. If it were not for the phase sensitivity measure $\Gamma_{N}$, one might classify it as a limit cycle as well. However, since it can be shown that the GOPY attractor has a fractal structure, we classify it as a strange nonchaotic attractor.

The final example given in this paper is the 3dimensional Lorenz attractor. Our computations show it to have a positive, a (near) zero, and a negative Lyapunov exponent. The positive exponent tells us the system is chaotic. According to Wolf et. al., the combination of positive, zero, and negative exponents is enough to classify it as strange [11]. The non-integer KaplanYorke dimension further strengthens this claim. When we study Figure 8 , it is interesting to note that at the two wings of the butterfly, orbits seem to be highly predictable. But when the orbits reach the mid-region of the butterfly, a chaotic mixing occurs. It is likely that this tearing property plays a major role in the Lorenz attractor's strangeness.

Often times it is the case that complex mathematical topics are best learned through example. It is for this reason that the various attractors and their classifications are provided in Section III. By investigating the distinguishing properties of nonstrange, strange nonchaotic, and strange chaotic attractors, we have hopefully gained some valuable insight into how chaotic dynamics and fractal geometries are connected.

\section{AcKNowledgements}

This project was part of a semester-long tutorial on chaos theory supervised by Dr. John David. Many thanks to him for all of his time and dedication to helping me come to grips with this complex topic.

All computations were done using GNU Octave - a free, high-level language similar to MATLAB.

\section{REFERENCES}

[1] R. Abraham and C. Shaw, Dynamics: The Geometry of Behavior (pt. 1), Ariel Press, 1985.

[2] J. P. Crutchiteld, J. D. Farmer, N. H. Packard, and R. S. Shaw, Chaos, Scientific American, 254 (1986), pp. 46-57.

[3] K. FALCONer, Fractal Geometry: Mathematical Foundations and Applications, John Wiley \& Sons, 1990.

[4] C. Grebogi, E. Ott, S. Pelikan, and J. A. Yorke, Strange attractors that are not chaotic, Physica D, 13 (1984), pp. 261-268.

[5] J. Milnor, On the concept of attractor, Communications in Mathematicals Physics, 99 (1985), pp. 177-195. 
[6] J. W. Milnor, Attractor. Scholarpedia, 2006. Accessed on $03 / 31 / 2010$.

[7] F. C. Moon, Chaotic and Fractal Dynamics: An Introduction for Applied Scientists and Engineers, John Wiley \& Sons, 1992.

[8] A. S. Pikovsky and U. Feudel, Characterizing strange nonchaotic attractors, Chaos, 5 (1995), pp. 253-260.

[9] A. Prasad, S. S. Negi, and R. Ramaswamy, Strange nonchaotic attractors, (2001).

[10] J. C. Sрrotr, Numerical calculation of largest lyapunov exponent, (2004). Accessed on 10/07/2010.

[11] A. Wolf, J. B. Swift, H. L. Swinney, and J. A. Vastano, Determining lyapunov exponents from a time series, Physica D, 16 (1985), pp. 285317. 